\title{
Clinicoradiological Features and Treatment Outcome of Supratentorial Intraparenchymal Epidermoid Cyst: A Report of Five Cases and Literature Review
}

\author{
Ashutosh Kumar ${ }^{1}$ Ved Prakash Maurya ${ }^{1}$ Soumen Kanjilal ${ }^{1} \quad$ Kamlesh Singh Bhaisora ${ }^{1}$ \\ Jayesh Sardhara ${ }^{1}$ Kuntal Kanti Das ${ }^{1}$ Anant Mehrotra' Arun Kumar Srivastava' \\ Awadhesh Kumar Jaiswal' Sanjay Behari ${ }^{1}$
}

${ }^{1}$ Department of Neurosurgery, Sanjay Gandhi Postgraduate Institute of Medical Sciences, Lucknow, Uttar Pradesh, India

\begin{abstract}
Address for correspondence Ved Prakash Maurya, MCh, MS, MBBS, Department of Neurosurgery, Sanjay Gandhi Postgraduate Institute of Medical Sciences, Lucknow, Uttar Pradesh 226014, India (e-mail: vpmsurgery@gmail.com).
\end{abstract}

J Neurosci Rural Pract 2021;12:571-580.

\begin{abstract}
Objectives Intraparenchymal epidermoid cysts (IECs) are rare lesions. They represent less than $1 \%$ of the intracranial epidermoid cysts. The supratentorial IEC is a clinically and prognostically distinct subset. Given the rarity, most of the articles are case reports. We present a series of five cases of supratentorial IEC to characterize their clinical presentation and outcome, with emphasis on the surgical features.

Materials and Methods We searched our database for all cases of intracranial epidermoid cysts operated between January 2005 and January 2020. Five patients were identified having IEC from the hospital information system and the neurosurgical operation record book. Standard craniotomy and decompression of the lesion were performed in all these patients. Standard postoperative care includes computed tomography scan of head on the day of surgery and magnetic resonance imaging of brain after 6 weeks to look for the residual lesion, if any. Subsequent follow-up visits in outpatient department to look for resolution of the presurgical symptoms.

Results The mean age of the patients in our series was 28.8 years (range: $28-40$ years.). All the five patients were male. Four patients had IEC involving frontal lobe and one in parietal lobe with a small occipital lobe extension. Seizure was the most common presenting complaint followed by headache. Complete excision was achieved in all the

Keywords

- intra-axial

- epidermoid cyst

- frontal lobe

- seizure

- headache cases. All the three patients with seizure attained seizure freedom postlesionectomy. Focal neurological deficits resolved gradually in postoperative period. There was no recurrence of lesion during follow-up.

Conclusion Supratentorial IEC most commonly affects young males, involve frontal lobe and present clinically with seizure. Complete surgical excision offers best outcome in the form of remission of seizure disorder.
\end{abstract}

published online May 11,2021
DOI https://doi.org/

$10.1055 / \mathrm{s}-0041-1730125$ ISSN 0976-3147 (c) 2021. Association for Helping Neurosurgical Sick People. This is an open access article published by Thieme under the terms of the Creative Commons Attribution-NonDerivative-NonCommercial-License, permitting copying and reproduction so long as the original work is given appropriate credit. Contents may not be used for commercial purposes, or adapted, remixed, transformed or built upon. (https://creativecommons.org/licenses/by-nc-nd/4.0/). Thieme Medical and Scientific Publishers Pvt. Ltd. A-12, 2nd Floor, Sector 2, Noida-201301 UP, India 


\section{Introduction}

Intracranial epidermoid cysts are rare congenital lesions characterized by a cystic lesion lined by stratified squamous epithelium, containing laminated keratin debris. These benign tumors are most commonly seen in cerebellopontine angle and account for 0.3 to $1.8 \%$ of the intracranial tumors. ${ }^{1}$ The other common locations include parapontine, parasellar, middle cranial fossa, diploe, and spinal canal. Epidermoid cysts that are purely within brain parenchyma are also known as "intra-axial," "intraparenchymal," or "intracerebral" epidermoid cysts. Supratentorial intraparenchymal epidermoid cyst (IEC) is rare with only 32 cases reported in literature. We present a series of five cases of supratentorial IEC and discuss the clinical presentation, radiological features, and surgical management along with review of literature.

\section{Materials and Methods}

\section{Patient Spectrum}

We searched the database of the department of neurosurgery, at a tertiary care referral center, for all cases of intracranial epidermoid cysts operated between January 2005 and January 2020. Details of surgical intervention and subsequent follow-up were extracted from our meticulous record keeping system.

\section{Inclusion Criteria}

1. Purely intraparenchymal epidermoid with no evidence of extension in the cisternal spaces.

2. Histologically proven primary IEC.

\section{Exclusion Criteria}

1. Recurrent or residual lesions

2. Intraparenchymal inclusion cysts other than epidermoid.

Five patients were identified to have purely intraparenchymal supratentorial epidermoid cyst. The preoperative magnetic resonance imaging (MRI), clinical findings, postoperative course, and follow-up details were retrieved from clinical record keeping system. The operative findings were noted from the hospital information system (HIS). Recent follow-up was done telephonically.

\section{Literature Review}

For literature review, the Medline database was searched through the PubMed. The search query entered was ("intraparenchymal"[All Fields] OR "intracerebral"[All Fields] OR "intraaxial"[All Fields]) AND ((((epidermoid cyst) OR (cholesteatoma)) OR (pearly tumor)) OR ("Epidermal Cyst"[Mesh])). Google Scholar was also searched with same keywords. Duplicate articles were removed. Articles published in English, with full-text available and having adequate information were included for further analysis.

\section{Statistical Methods}

Statistical analysis was done using Statistical Package for the Social Sciences (SPSS) version 22.00 (IBM, New York, United States). The parametric data were expressed as mean/median and nonparametric data were expressed as number ( $n$ ) and percentage.

\section{Results}

Five pathologically confirmed cases of intracranial epidermoid cyst were identified that were confirmed to be intraparenchymal based on the radiological and intraoperative findings. All the patients were young males with mean age of 28.8 years (range: $28-40$ years.). Four had IEC in frontal lobe and one in parietal lobe with a small occipital lobe extension. Three patients presented with generalized tonic-clonic seizures. Two patients had features of raised intracranial pressure (ICP) and two patients complained of localized headache ipsilateral to the lesion in the frontal region. Complete excision was achieved in all cases with no significant postoperative complications. The seizure outcome postsurgery was also good. None of the patients developed postoperative aseptic meningitis or recurrence at subsequent follow-up visits.

The literature search for supratentorial IEC identified 23 articles reporting 32 cases meeting the selection criteria. These cases along with the cases in present series are summarized in -Table 1.2-24 Of these 37 cases, $62.2 \%(n=23)$ patients were males and $37.8 \%$ were females. The mean age of the patients was 37.4 years (standard deviation \pm 15.5 ). The lesions were reported in frontal (54.1\%), temporal (32.4\%), parietal lobe (10.8\%), and thalamus (2.7\%) in decreasing order of frequency of their occurrence. On MRI, 93.1\% $(n=27 / 29)$ showed diffusion restriction irrespective of T1 or T2 findings. Eleven (37.9\%) cases showed significant enhancement upon administration of contrast agent. Total excision was achieved in $86.7 \%$ patients $(n=26)$. Most of the patients recovered uneventfully. However, the reported complications included transient weakness, third nerve dysfunction, cerebrospinal fluid leak, hemianopsia, aseptic meningitis, and central retinal artery occlusion. Most of the complications were related to lobar involvement of brain, while others were related to nonresolution of raised ICP. Mortality was reported in one case following recurrence; $95.65 \%$ cases reported complete freedom from seizure disorder postlesionectomy.

\section{Illustrative Case Presentation}

A 28-year-old man presented with multiple episodes of sleep onset generalized tonic-clonic seizures for 3 years. There were no focal neurological deficits. The seizures were controlled with two antiepileptic drugs (AEDs). The MRI was suggestive of a $4 \times 3 \times 3 \mathrm{~cm}$ intra-axial lesion involving the left parieto-occipital lobe. Local mass effect resulting in flattening of gyri and obliteration of sulci was seen. The lesion was hypointense on $\mathrm{T} 1$ and hyperintense on T2-weighted imaging. There was no perilesional edema and no contrast enhancement. Diffusion restriction was present on diffusion-weighted imaging (DWI) sequence (-Fig. 1). A preoperative diagnosis of IEC was made.

Patient was given the antiepileptics and injection dexamethasone $8 \mathrm{mg}$ intravenous (IV) along with 
Supratentorial Intraparenchymal Epidermoid Cyst: A Report of Five Cases and Literature Review Kumar et al. 573

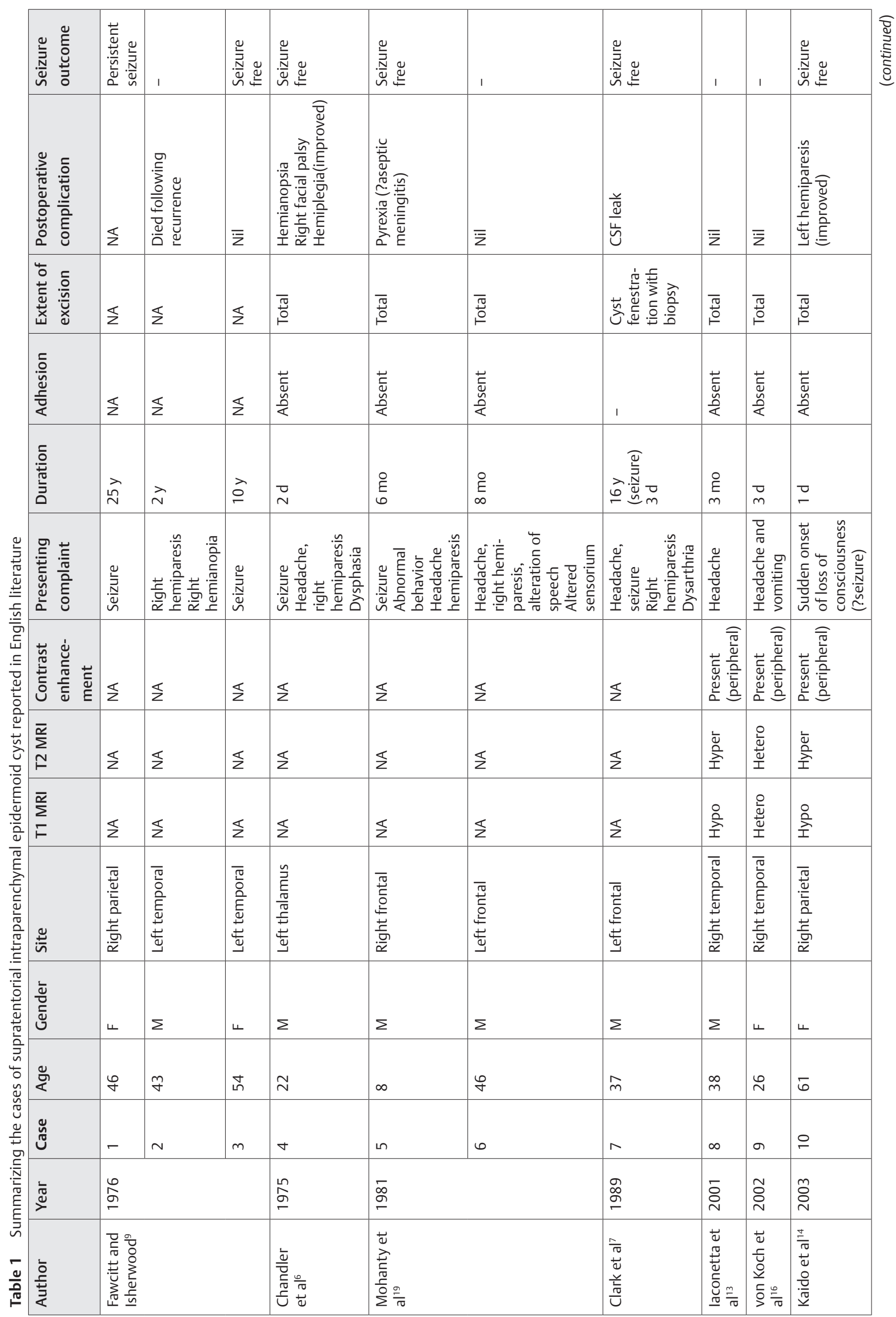




\begin{tabular}{|c|c|c|c|c|c|c|c|c|c|c|c|c|c|c|c|c|}
\hline 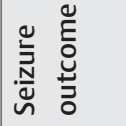 & 岕 & 1 & 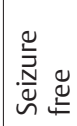 & 岕 & $\mathbb{z}$ & $\Sigma$ & 总 & لّ & 岕 & 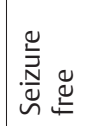 & 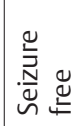 & 訔 & 离 & 1 & 莺 & 岕 \\
\hline 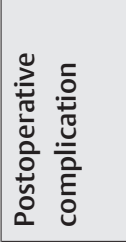 & $\overline{\bar{z}}$ & $\overline{\bar{z}}$ & 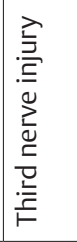 & $\overline{\bar{z}}$ & $\S$ & $\S$ & $\overline{\bar{z}}$ & $\overline{\bar{z}}$ & 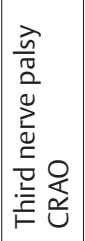 & $\overline{\bar{z}}$ & $\overline{\bar{z}}$ & $\overline{\bar{z}}$ & $\overline{\bar{z}}$ & $\overline{\bar{z}}$ & $\overline{\bar{z}}$ & $\overline{\bar{z}}$ \\
\hline 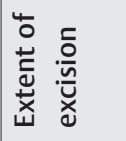 & $\begin{array}{l}\overline{\widetilde{\pi}} \\
\stackrel{0}{\circ}\end{array}$ & $\Sigma$ & $\begin{array}{l}\overline{\sqrt{n}} \\
\stackrel{0}{0}\end{array}$ & $\begin{array}{l}\overline{\widetilde{\pi}} \\
\stackrel{0}{0}\end{array}$ & $\Sigma$ & 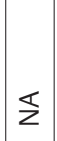 & 吾 & 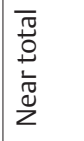 & $\begin{array}{l}\overline{\widetilde{\pi}} \\
\stackrel{0}{0}\end{array}$ & $\begin{array}{l}\overline{\widetilde{0}} \\
\stackrel{0}{\circ}\end{array}$ & $\begin{array}{l}\overline{\widetilde{\pi}} \\
\stackrel{0}{0}\end{array}$ & 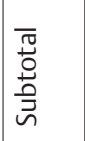 & 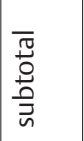 & $\begin{array}{l}\overline{\sqrt{n}} \\
\stackrel{0}{0}\end{array}$ & $\begin{array}{l}\overline{\widetilde{\pi}} \\
\stackrel{0}{0}\end{array}$ & $\begin{array}{l}\overline{\widetilde{n}} \\
\stackrel{0}{\circ}\end{array}$ \\
\hline $\begin{array}{l}\frac{.}{0} \\
\frac{0}{4} \\
\frac{5}{2}\end{array}$ & $\begin{array}{l}\overrightarrow{\tilde{v}} \\
\text { } \\
\hat{\alpha}\end{array}$ & $\S$ & $\Sigma$ & 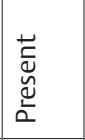 & $\S$ & $\Sigma$ & 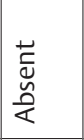 & 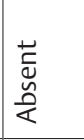 & 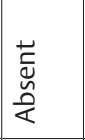 & 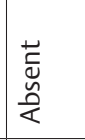 & 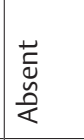 & 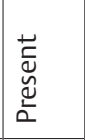 & 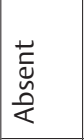 & 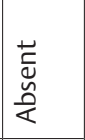 & 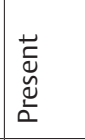 & 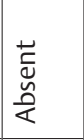 \\
\hline 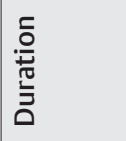 & $\stackrel{\stackrel{Q}{\mathfrak{V}}}{\sim}$ & 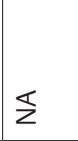 & 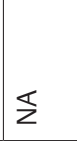 & 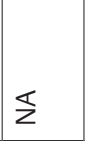 & $\Sigma$ & $\Sigma$ & E̊ & बे & $\Sigma$ & $\begin{array}{l}\stackrel{0}{E} \\
\infty\end{array}$ & $\Sigma$ & 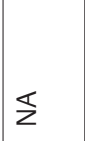 & $\Sigma$ & $\underset{\stackrel{\text { }}{\mathrm{V}}}{\mathrm{N}}$ & है & $\bar{m}$ \\
\hline 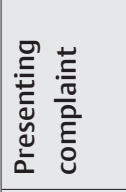 & 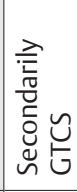 & 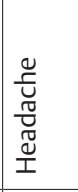 & 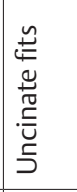 & 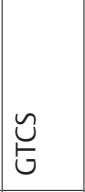 & $\Sigma$ & $\Sigma$ & 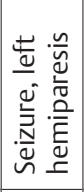 & 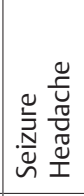 & $\breve{U}$ & 气 & 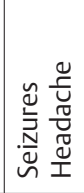 & $\breve{气}$ & 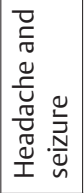 & 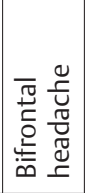 & 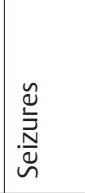 & $\stackrel{\breve{v}}{\breve{v}}$ \\
\hline 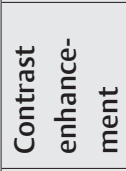 & $\overline{\bar{z}}$ & 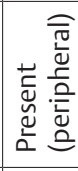 & 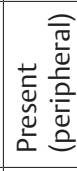 & $\overline{\bar{z}}$ & $\overline{\bar{z}}$ & $\overline{\bar{z}}$ & $\overline{\bar{z}}$ & $\overline{\bar{z}}$ & $\overline{\bar{z}}$ & $\overline{\bar{z}}$ & $\overline{\bar{z}}$ & $\overline{\bar{z}}$ & $\overline{\bar{z}}$ & 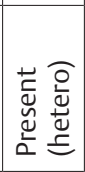 & 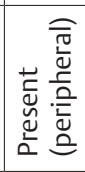 & 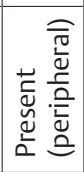 \\
\hline 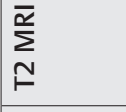 & 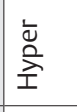 & 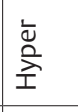 & \begin{tabular}{|l} 
\\
\multirow{2}{*}{} \\
$\frac{0}{I}$ \\
\end{tabular} & 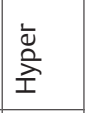 & 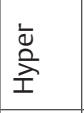 & 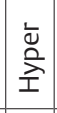 & 咅 & 乏 & $\Sigma$ & $\stackrel{\circ}{\stackrel{2}{x}}$ & 号 & 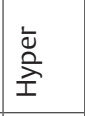 & 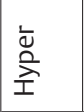 & 号 & 离 & 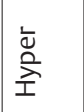 \\
\hline $\begin{array}{l}\overline{\text { r }} \\
F\end{array}$ & 斑 & 号 & 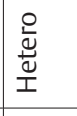 & 옷 & $\stackrel{\circ}{\stackrel{\circ}{x}}$ & 高 & 号 & 옷 & 亲 & 㐫 & 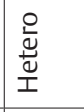 & 只 & $\stackrel{\circ}{\stackrel{\circ}{\text { I }}}$ & $\begin{array}{l}\text { ऐo } \\
\text { 全 }\end{array}$ & 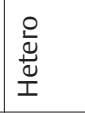 & 竞 \\
\hline$\stackrel{\oplus}{*}$ & 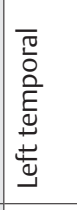 & 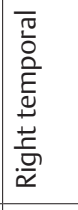 & 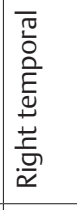 & 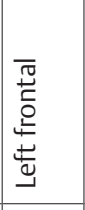 & 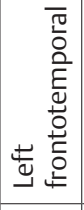 & 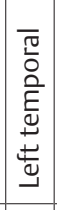 & 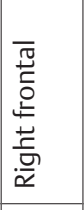 & 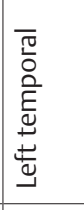 & 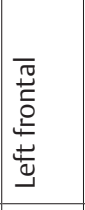 & 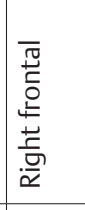 & 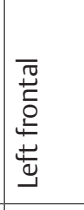 & 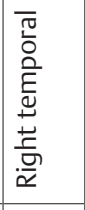 & 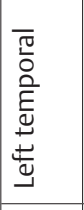 & 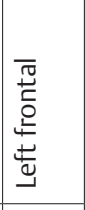 & 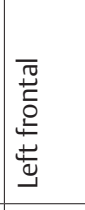 & 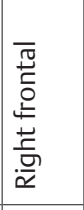 \\
\hline 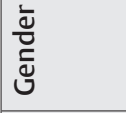 & 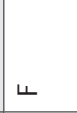 & 4 & $\Sigma$ & $\Sigma$ & $\Sigma$ & 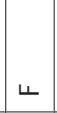 & $\Sigma$ & 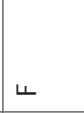 & $L$ & $\Sigma$ & $\Sigma$ & 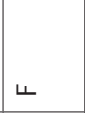 & \llcorner & \llcorner & $\Sigma$ & $\Sigma$ \\
\hline 芩 & $\stackrel{0}{2}$ & 우 & 5 & 우 & $\mathscr{f}$ & $\stackrel{\infty}{+}$ & 6 & 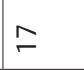 & $\simeq$ & 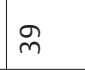 & 约 & $\stackrel{\Omega}{\longrightarrow}$ & 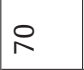 & 으 & $\stackrel{*}{m}$ & $\simeq$ \\
\hline 岕 & $\mp$ & 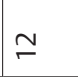 & $\stackrel{m}{r}$ & $\stackrel{\nabla}{\sqsubset}$ & $\stackrel{n}{\leftarrow}$ & $\div$ & 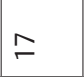 & $\stackrel{\infty}{-}$ & $\stackrel{\sigma}{ }$ & $\stackrel{\sim}{N}$ & $\bar{\sim}$ & $\approx$ & $\ddot{\sim}$ & $\stackrel{ \pm}{\sim}$ & $\stackrel{\Perp}{\sim}$ & $\stackrel{\sim}{\sim}$ \\
\hline 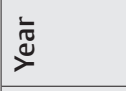 & ণ্ণ & 足 & $\stackrel{\infty}{\stackrel{\infty}{े}}$ & ¿̊̀ & $\stackrel{\infty}{\stackrel{\infty}{\sim}}$ & & ஓे & ஓे & & $\stackrel{0}{\stackrel{2}{N}}$ & 竝 & $\overline{\grave{N}}$ & & 水 & $\stackrel{\infty}{\stackrel{\infty}{\nu}}$ & $\stackrel{\infty}{\stackrel{\infty}{N}}$ \\
\hline 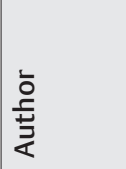 & 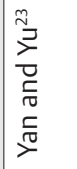 & $\begin{array}{l}\frac{\pi}{2} \\
\stackrel{\Sigma}{\Sigma} \\
\tilde{\Sigma}\end{array}$ & 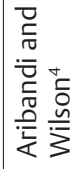 & 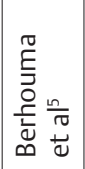 & $\begin{array}{l}\frac{\sqrt{2}}{\sqrt{0}} \\
\stackrel{0}{0} \\
\overrightarrow{\underline{1}}\end{array}$ & & $\begin{array}{l}\frac{0}{\pi} \\
\frac{\pi}{0} \\
\frac{0}{0} \\
0\end{array}$ & 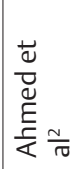 & & 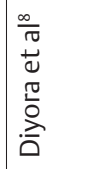 & 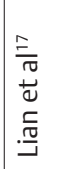 & 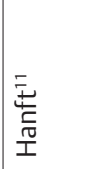 & & 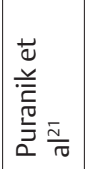 & 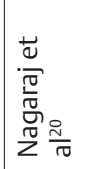 & 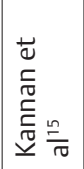 \\
\hline
\end{tabular}


Supratentorial Intraparenchymal Epidermoid Cyst: A Report of Five Cases and Literature Review Kumar et al. 575

\begin{tabular}{|c|c|c|c|c|c|c|c|c|c|c|c|c|}
\hline 离 & 1 & 䓂 & , & $1 \frac{\Sigma}{z}$ & $\underline{z}$ & 产 & 离 & 焉 & , & 1 & 产苞芯 & 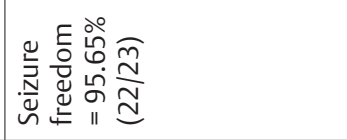 \\
\hline 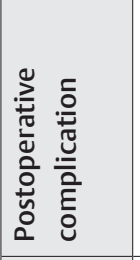 & $\overline{\bar{z}}$ & $\overline{\bar{z}}$ & $\overline{\bar{z}}$ & $\overline{\bar{z}} \bar{z}$ & 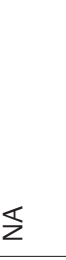 & $\overline{\bar{z}}$ & $\overline{\bar{z}}$ & $\overline{\bar{z}}$ & 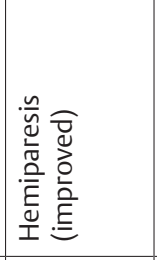 & $\overline{\bar{z}}$ & 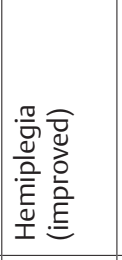 & 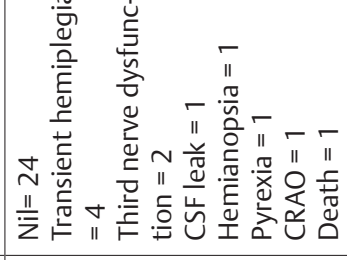 \\
\hline 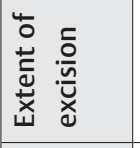 & $\begin{array}{l}\bar{\pi} \\
\stackrel{\pi}{0} \\
\stackrel{0}{\circ}\end{array}$ & 苞 & 丞 & 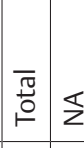 & ż & 苟 & 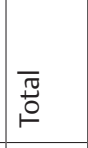 & $\begin{array}{l}\text { 吾 } \\
\stackrel{\circ}{\circ}\end{array}$ & $\begin{array}{l}\text { 嵒 } \\
\stackrel{\circ}{\circ}\end{array}$ & 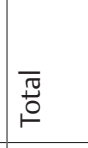 & 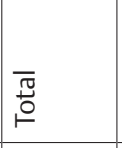 & 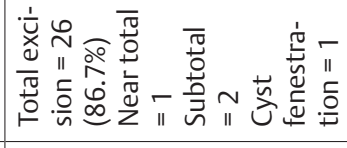 \\
\hline 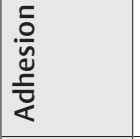 & 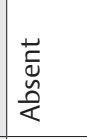 & 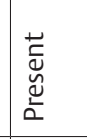 & \begin{tabular}{|l} 
\\
蒂 \\
商
\end{tabular} & 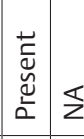 & 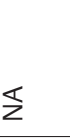 & 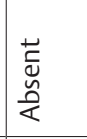 & 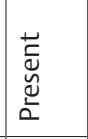 & 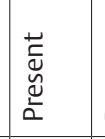 & 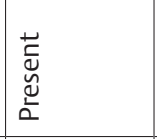 & 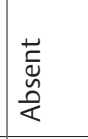 & 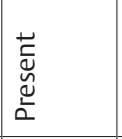 & 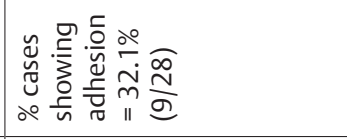 \\
\hline 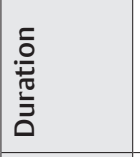 & $\Sigma$ & $\frac{\pi}{z}$ & $\bar{z}$ & $\Sigma 0$ & 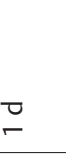 & $\frac{\pi}{z}$ & $\vec{m}$ & $\geq$ & 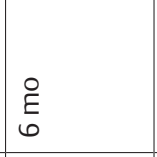 & $\underset{\infty}{\stackrel{\bigcirc}{E}}$ & $\geq$ & 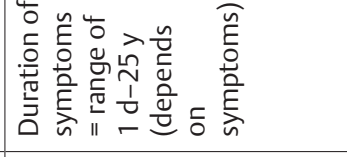 \\
\hline 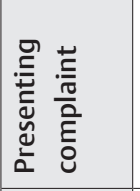 & 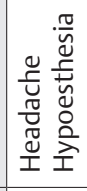 & 岕 & 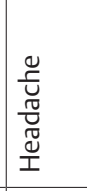 & 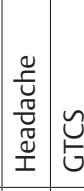 & 气 & $\breve{U}$ & 气 & 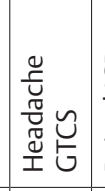 & 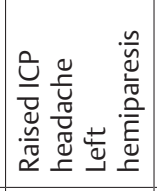 & 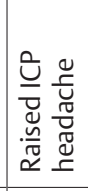 & 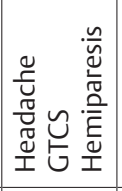 & 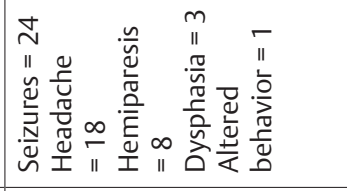 \\
\hline 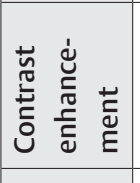 & $\overline{\bar{z}}$ & $\overline{\bar{z}}$ & 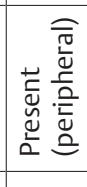 & $\overline{\bar{z}} \frac{\bar{z}}{z}$ & $\Sigma$ & $\overline{\bar{z}}$ & $\overline{\bar{z}}$ & 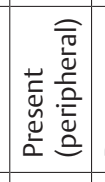 & 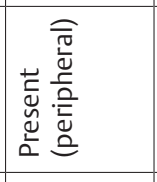 & $\overline{\bar{z}}$ & $\overline{\bar{z}}$ & 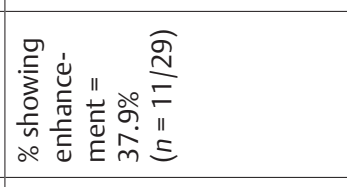 \\
\hline$\sum_{\tilde{N}}^{\bar{N}}$ & 㕍 & 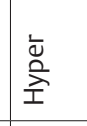 & 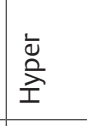 & 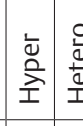 & 这 & 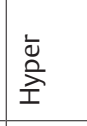 & 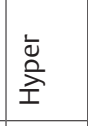 & 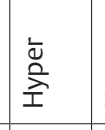 & 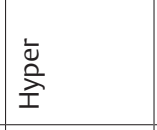 & 袲 & 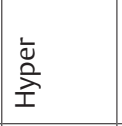 & 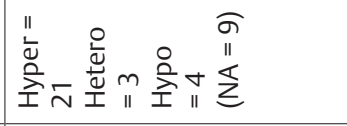 \\
\hline $\begin{array}{l}\overline{\underline{e}} \\
F \\
F\end{array}$ & 올 & 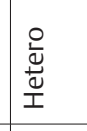 & 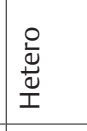 & 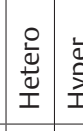 & 厗 & $\stackrel{\circ}{\grave{1}}$ & 景 & $\stackrel{\circ}{\circ}$ & 咅 & 离 & 豙 & " \\
\hline ڤั & 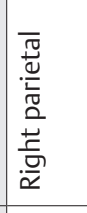 & 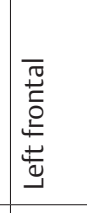 & 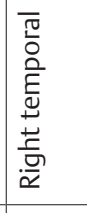 & 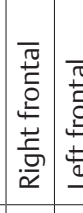 & 焉 & 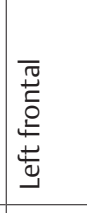 & 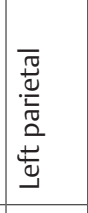 & 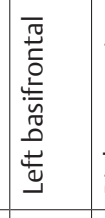 & 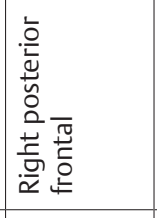 & 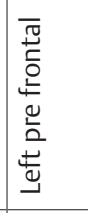 & 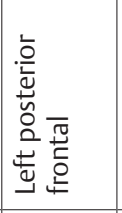 & 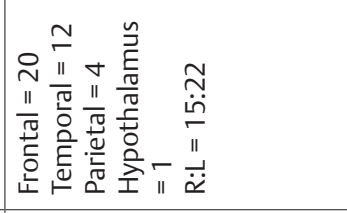 \\
\hline Uूँ & 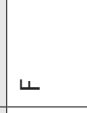 & $\Sigma$ & $\Sigma$ & $\Sigma u$ & 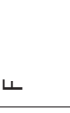 & $\Sigma$ & $\Sigma$ & $\Sigma$ & $\Sigma$ & $\Sigma$ & $\Sigma$ & 崖 \\
\hline 茕 & ซ & $\stackrel{m}{m}$ & F & in 6 & 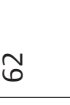 & \& & $\stackrel{\infty}{\sim}$ & $\stackrel{\infty}{.}$ & q & $\stackrel{\infty}{\sim}$ & $\stackrel{\circ}{m}$ & 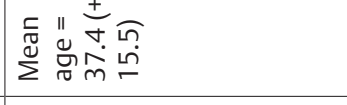 \\
\hline 苂 & $\grave{N}$ & $\stackrel{\infty}{\sim}$ & I & $\stackrel{m}{\bar{m}}$ & $\bar{m}$ & $\approx$ & m & 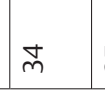 & $\stackrel{\leftrightarrow}{m}$ & m & $\hat{m}$ & 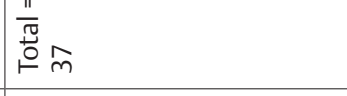 \\
\hline 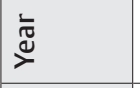 & $\stackrel{\infty}{\grave{n}}$ & & & & ì & ָิ & & & & & & $\gtrsim$ \\
\hline ఏ & 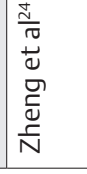 & & & & 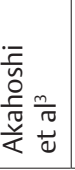 & 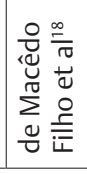 & 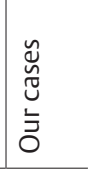 & & & & & 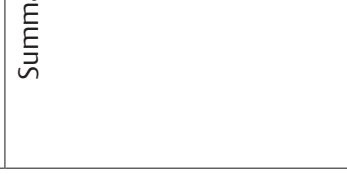 \\
\hline
\end{tabular}




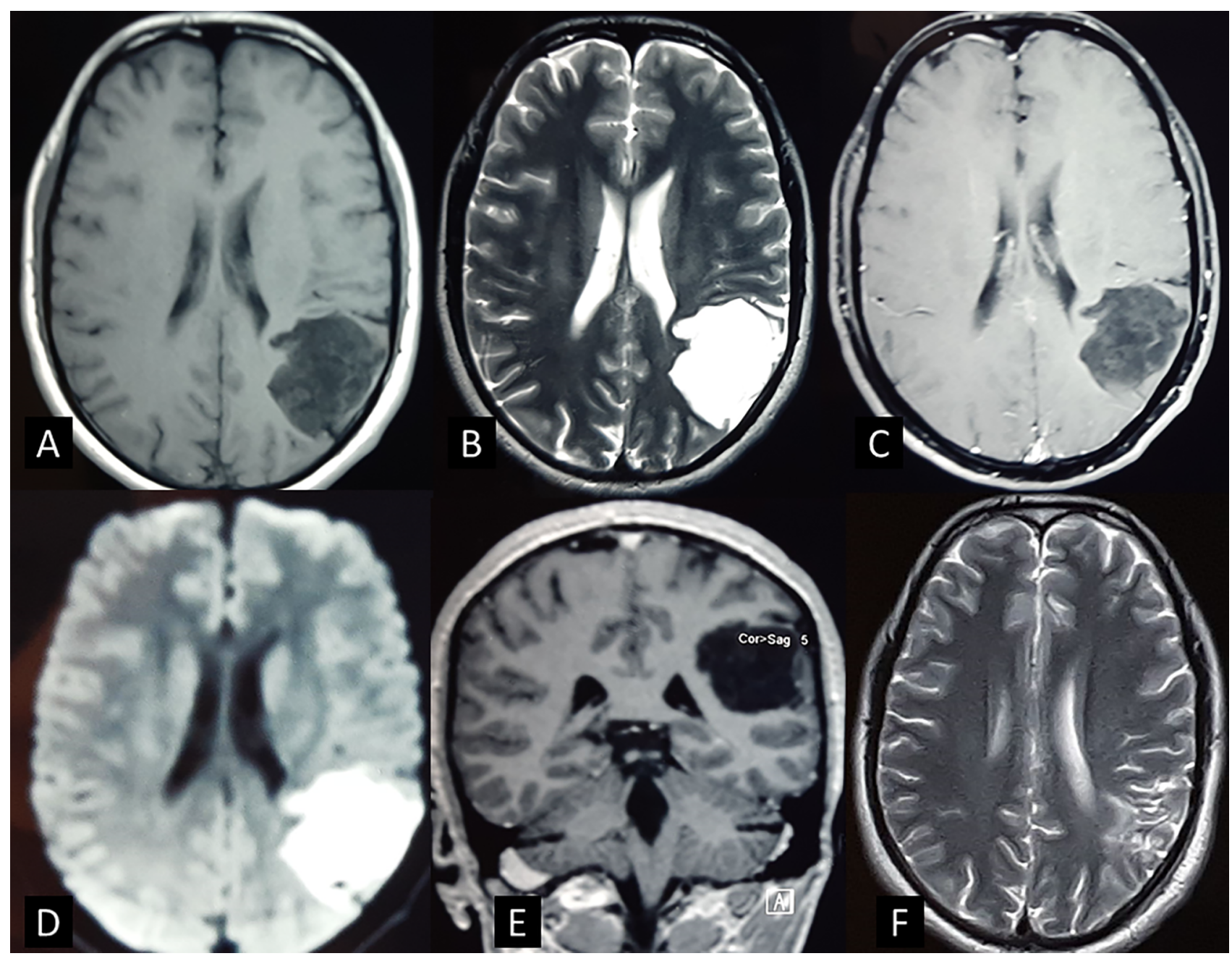

Fig. 1 The magnetic resonance imaging of patient (33). (A) The lesion is hypointense on T1-weighted axial image and (B) hyperintense on T2-weighted image. (C) There is no contrast enhancement seen on T1 + contrast axial image. (D) The lesion is seen hyperintense of diffusion-weighted imaging. (E) T1-weighted coronal image: The lesion is involving the left parietal lobe and has thin layer of brain parenchyma covering it (completely intraparenchymal). (F) Postoperative T2-weighted image showing complete tumor excision.

preoperative medication. A left parasagittal parietal craniotomy was performed. The dura was reflected medially. The brain parenchyma overlying the tumor was pale yellow and the postcentral gyri seemed widened. A $1 \times 1 \mathrm{~cm}$ corticectomy was done and tumor was reached at a depth of $1 \mathrm{~cm}$. The tumor was well defined, lobulated, capsulated, pearly white, and avascular. There was keratinized debris with no hair or calcification. The capsule at places was adherent to the brain parenchyma. Rest of the tumor had good plane and could be easily excised completely. Care was taken to avoid spillage. Adequate wash with warm saline was done postresection. Intraoperative findings were confirmatory of IEC (-Fig. 2). Postoperatively, patient recovered well with no focal neurological deficits or seizures. Dexamethasone was given in tapering dose for 3 days. Same AEDs were continued. Patient was discharged on postoperative day 5. The AEDs were tapered off in follow-up after 6 months. At 2 years follow-up, patient is having no seizures and all AEDs have been stopped. Histopathology of the lesion revealed epidermoid cyst with characteristic appearances ( - Fig. 3 ).

\section{Discussion}

Intracranial epidermoid cyst is postulated to develop from the embryonic cell resets or from the sequestrated ectodermal cell rests during third and fifth weeks of intrauterine life. ${ }^{6,13,25,26}$ IEC is also supposed to result from the same pathogenesis with the final location possibly depending on the timing of sequestration..$^{14}$ These lesions represent less than $1 \%$ of intracranial epidermoid cysts. ${ }^{17}$ They have been reported in both supra- and infratentorial locations. The infratentorial locations include the cerebellum and the brain stem. Despite similar pathogenesis, epidermoid cysts in these two locations differ clinically and the management principles also differ. An aggressive resection in pontine IEC may have adverse outcome, while a subtotal resection for supratentorial lesions is associated with increased postoperative complications and recurrence. This article focuses on the clinical characteristics, surgical principles, and outcome of the supratentorial lesions. All of the previous articles and reviews have analyzed these heterogenous groups together. 


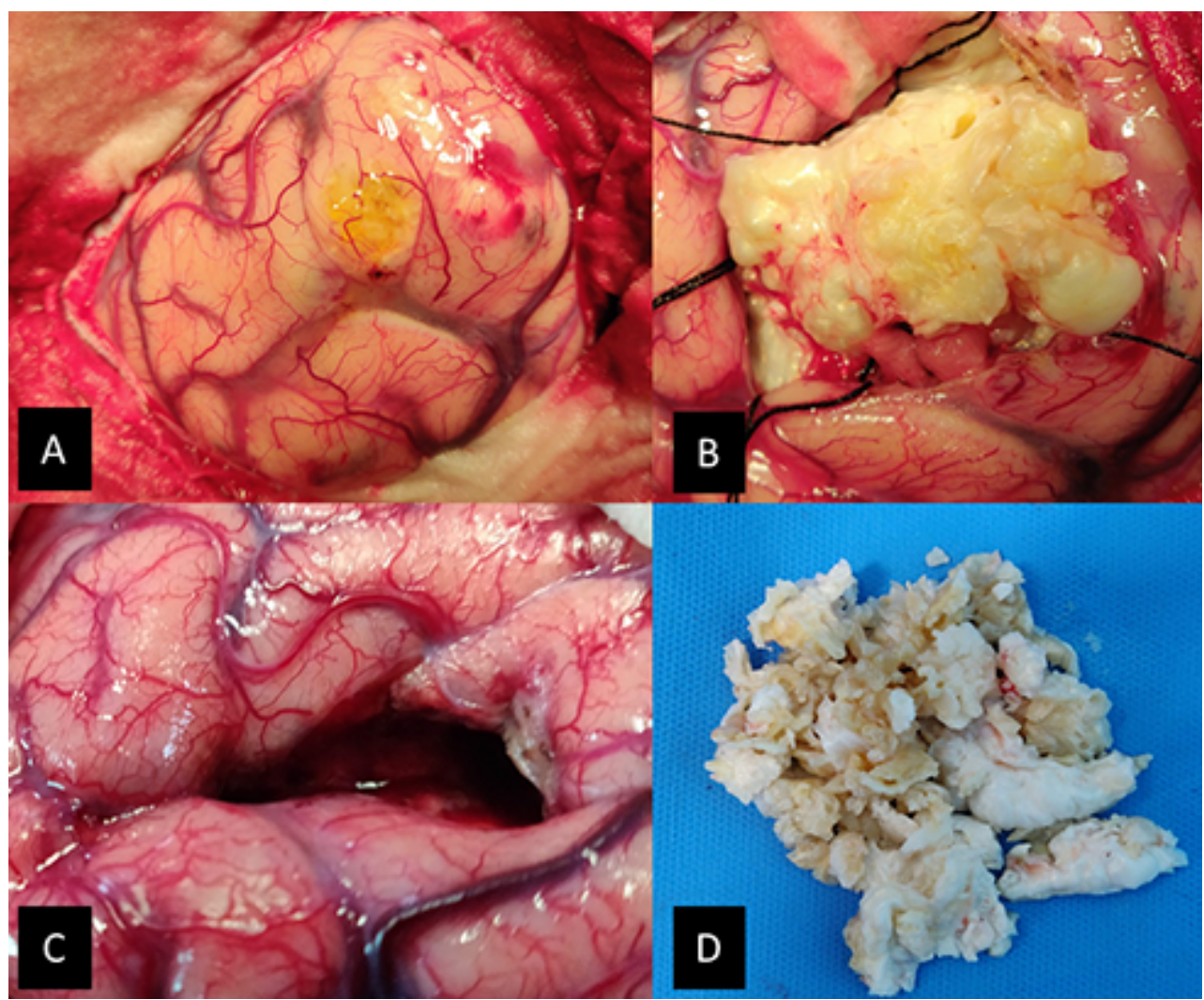

Fig. 2 The intraoperative images of the same patient (33). (A) The overlying cortex was yellowish. The surroundings were covered with cotton patties to prevent contamination with the tumor debris. (B) The tumor had a yellowish hue and good plane with brain parenchyma. (C) The tumor was completely excised with the tumor capsule. (D) Tumor was "pearly." It was removed in piecemeal through a small corticectomy.

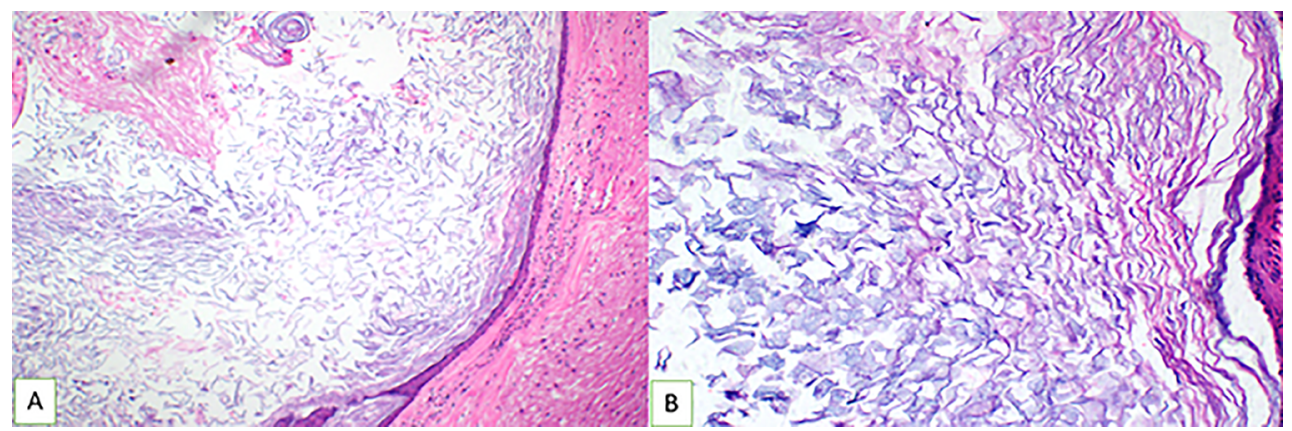

Fig. 3 Hematoxylin and eosin-stained sections showing a cyst wall lined by stratified squamous epithelium and keratin flakes in the lumen. Adjacent compressed cerebral parenchyma is seen at the periphery (A) (A: Original magnification $\times 10$, B: original magnification $\times 20$ ).

\section{Clinical Presentation}

The present series and the review of literature suggest that men are affected more than women by supratentorial IEC $(\mathrm{M}: \mathrm{F}=1.6: 1)$. Most of these patients are of young or middle age and present with seizures as the most common complaint. Headache is also a common presenting complaint. Other symptoms include focal neurological deficits, meningitis, altered sensorium, and lobar signs such as disinhibition, personality changes, distractibility, etc. Zheng et al in their series of six patients had four patients in supratentorial compartment. ${ }^{24}$ There were equal males and females, and headache was the most common presenting complaint. Some patients can present acutely with feature of raised
ICP following increased perilesional edema or hemorrhage ( - Fig. 4) $)^{7,10,11,23}$ We found frontal lobe to be the most common site of supratentorial IEC (54.1\%). The other sites included temporal lobe, parietal lobe, and thalamus. Cases of IEC involving corpus callosum, pineal gland, and occipital lobe have been also reported. ${ }^{14}$ Most of these reports are from Japan. Kaido et al reported temporal lobe as the most common site of IEC in the Brain Tumor Registry of Japan and in their literature review. ${ }^{14}$ These features are significantly different from the more common cisternal epidermoid cysts. They present most commonly in cerebellopontine cistern (60\% of all epidermoid cysts) with headache or multiple cranial nerve palsy. 


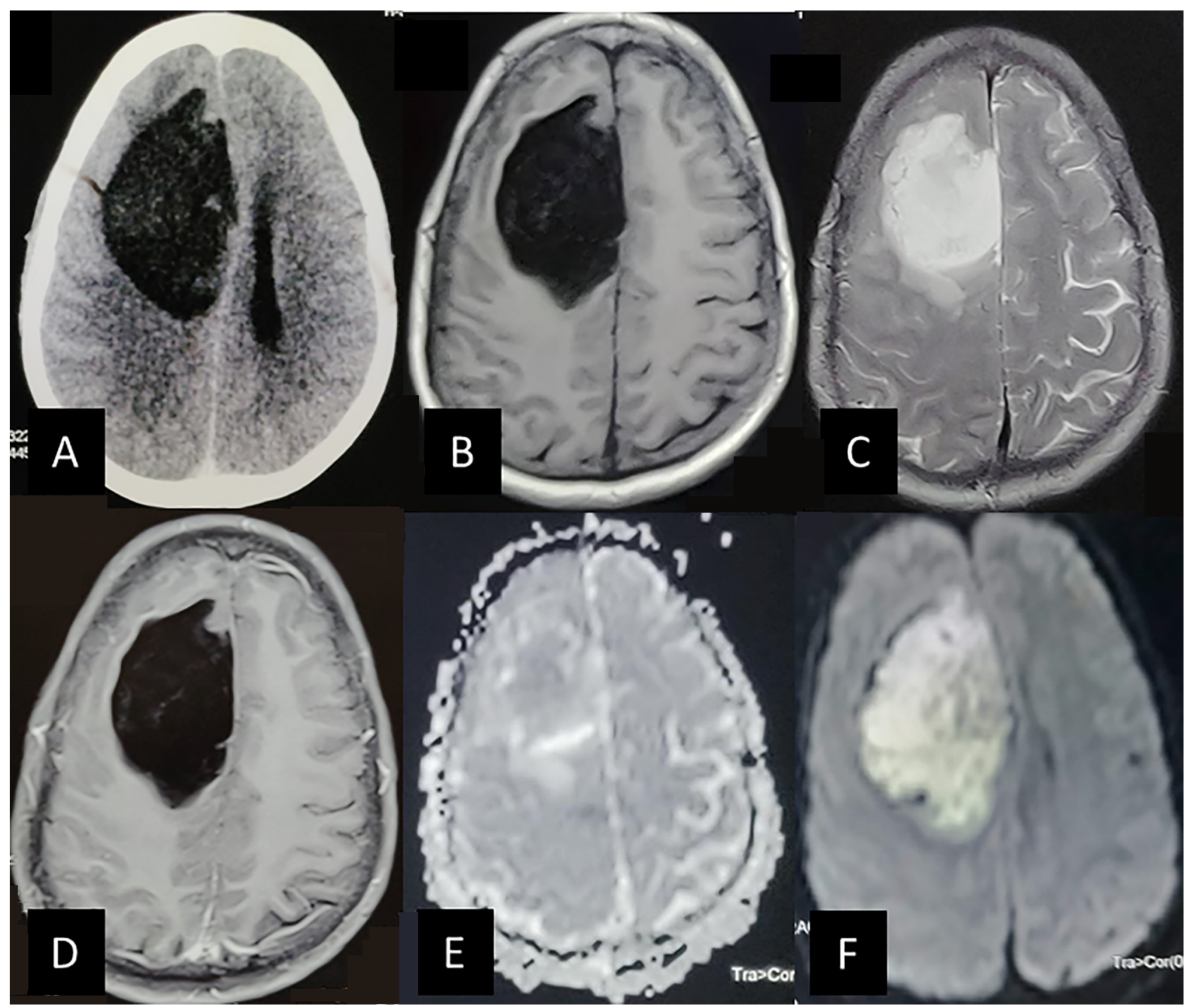

Fig. 4 The magnetic resonance imaging of the patient (35). Patient had presented with raised intracranial pressure headache and progressive hemiparesis. (A) The tumor is hypodense on plain computed tomography of the head involving the right frontal lobe. Specks of hyperdensity is seen with the tumor. It is compressing the ipsilateral lateral ventricle. (B) Tumor is hypointense on T1 axial image with tiny specks of hyperintensity within. (C) The tumor is homogenously hyperintense in T2- weighted images. The compression and flattening of gyri is appreciated. (D) There is no contrast enhancement seen on T1 + contrast image. (E) It is isointense on apparent diffusion coefficient sequence and (F) hyperintense on the diffusion-weighted imaging sequence. These findings are suggestive of intraparenchymal epidermoid cyst with possible small hemorrhages (late subacute). No attachment to the falx was noted intraoperatively.

\section{Radiological Features}

The contents of ruptured cysts produce local inflammation. There is development of surrounding gliosis and granulation tissue formation that accounts for hemorrhage and peripheral contrast enhancement. ${ }^{10,27,28}$ Irrespective of other MRI sequences, the hyperintense signal on DWI is diagnostic of IEC and also recommended for assessing extent of excision postoperatively. The DWI contrast depends on the apparent diffusion coefficient (ADC) value and the proton density. IEC has a low ADC due to the cystic nature of the tumor along with remarkable $\mathrm{T} 2$ prolongation causing $\mathrm{T} 2$ shine through effect. The histopathological finding responsible for the hyperintense signal on DWI is the presence of abundant keratin debris filled in the lumen of the cyst. The restriction of water diffusion is due to the concentric layers of keratin filaments within the cyst, thus limiting the movement of water molecules to planes formed between the layers of keratin. ${ }^{29}$ However, sometimes there may be hypointense signal on DWI. ${ }^{17,21}$

An absence of contrast enhancement is not a reliable diagnostic criterion for IEC as $37.9 \%$ cases showed some enhancement. However, the pattern was always peripheral. Contrast enhancement has been reported in 35\% of epidermoid cysts which is comparable to IEC. ${ }^{30}$ The cisternal and IEC share similar MRI findings. Peritumoral edema and calcification may also be present in few cases., ${ }^{4,10,14,15,20} \mathrm{Few}$ cases of "white epidermoid cysts" has also been reported. These cysts are T1 hyperintense due to predominant lipid content. ${ }^{8,21}$ Such atypical MRI features are common in IECs. ${ }^{17}$ It is commonly misdiagnosed as ganglioglioma (in presence of calcification) or cerebral abscess (in presence of thin peripheral enhancement) based on preoperative MRI findings. 


\section{Surgical Features}

Most of the patients undergoing surgery had complete excision of the lesion. The tumor was well capsulated and had good plane in the form of well-defined tumor-brain interface with gliotic changes in the adjacent brain parenchyma, facilitating complete tumor excision. There is a thin layer of gliotic brain parenchyma, with variable thickness, surrounding the tumor which helps in demarcating the lesion. ${ }^{5,19}$ However, most of them had some degree of adhesion requiring careful dissection during surgery. Intraoperative spillage can be prevented by using large cotton patties spread around the site of tumor excision. Tumor can be visualized after a small corticectomy. Further steps of tumor decompression must be performed under high magnification of surgical microscope with copious irrigation of saline over the operative field. In majority of the cases, the tumor gets delivered with small-to-moderate-sized corticectomy on performing sequential decompression followed by dissection, with identification of the tumor-brain interface all around. There is available literature supporting the adherence of the dura mater to the underlying tumor., ${ }^{5,11,20}$ Intraoperative monitoring is usually not required as the anatomical landmarks are well preserved and tumor-brain interface is well defined. Because of their extremely slow growth, these tumors tend to push the adjacent brain parenchyma along with the eloquent cortex rather than infiltrating/encasing the neural structures such as the cisternal epidermoid cyst. This leads to a significant amount of disparity between the actual location of the eloquent regions and their expected location based on the craniometric points. The clinical symptoms are mostly due to the local irritation or the compression by the tumor on the adjacent brain parenchyma. de Macêdo Filho et al reported a case of IEC near Broca's area. The tumor could be completely excised under awake craniotomy. ${ }^{18}$ These symptoms tend to gradually resolve completely following surgery. ${ }^{10,14,19}$ However, aggressive resection of adherent capsule on the eloquent area may injure it leading to permanent deficit. Few authors recommend safe decompression as goal of surgery in eloquent zones. ${ }^{5,13}$ Most of the authors reported a yellowish tint to the tumor. This could be suggestive of chronic microhemorrhages around the tumor..$^{14}$ Adequate intraoperative saline wash and steroid cover may prevent postoperative aseptic meningitis and hydrocephalus. Some authors recommend irrigation of surgical field with hydrocortisone. ${ }^{31}$ We had not used it in any of our cases. Chemical meningitis was not reported in any of the cases. Eight $\mathrm{mg}$ IV dexamethasone before induction and $4 \mathrm{mg}$ IV dexamethasone TDS (three times daily) for 3 days postoperatively were given in all these cases. Intraoperative spillage of cyst content must be minimized. Unlike the cisternal cysts that are associated with chemical meningitis, we found both in our experience and on literature review that its incidence is less frequently reported with IEC. This is possibly due to less chances of spillage of cyst contents within the subarachnoid space in parenchymal cysts.

\section{Clinical Course and Outcome}

Adequate dose of AED in preoperative period and continuation in immediate postoperative period is helpful in preventing any seizure episodes. Most of the patients recover uneventfully. The possible postoperative complications include hemorrhage, aseptic meningitis, and wound-associated complications. Recurrence is common if subtotal excision was performed. However, there exist long duration of symptom free period postexcision before patient develop symptoms due to recurrence. The seizure outcome is good with most patients attaining seizure freedom. ${ }^{5,17,20}$

\section{Supratentorial versus Infratentorial IEC}

These two subgroups differ significantly by virtue of their location. The infratentorial locations include the brain stem and the cerebellum. All the reported cases of pediatric infratentorial IEC are in brain stem. More females were affected than males. ${ }^{24}$ These tumors present with hemiparesis, seventh nerve palsy, sixth nerve palsy, and gait ataxia. ${ }^{32}$ The radiological features of these two groups are comparable. As against supratentorial IEC, the principle of surgery in brain stem IEC is safe decompression. Most authors reported adverse outcome with aggressive resection. ${ }^{33,34}$ Mortality has been reported in three cases of brain stem IEC against only one in supratentorial IEC. Overall, supra- and infratentorial IECs are distinct clinical entities requiring different approach for management.

\section{Limitations}

This is a retrospective study. Given the small sample size, the results of this study cannot be generalized. However, being a subset of a rare pathology, a larger sample size is less feasible. Meticulous archival of surgical findings in HIS for record keeping was helpful in avoiding recall bias. Further reporting of such cases would benefit in better understanding this uncommon neurosurgical entity.

\section{Conclusion}

The most common presenting complaint is seizure. Frontal lobe has the maximum predilection for the occurrence of supratentorial IEC. Males are affected more often than female population. The aim of surgery should be gross total excision which can be achieved in most cases. The focal neurological deficits gradually resolve postoperatively. Complete seizure control is achievable with lesionectomy alone. Precautions must be taken against postoperative seizures and aseptic meningitis. The overall outcome is good in view of benign nature of the disease.

\section{Note}

This study was presented at the Department of Neurosurgery, Sanjay Gandhi Postgraduate Institute of Medical Sciences, Lucknow, December 1, 2020.

\section{Funding \\ None.}

\section{Conflict of Interest}

None declared. 


\section{References}

1 Yamakawa K, Shitara N, Genka S, Manaka S, Takakura K. Clinical course and surgical prognosis of 33 cases of intracranial epidermoid tumors. Neurosurgery 1989;24(4):568-573

2 Ahmed I, Auguste KI, Vachhrajani S, Dirks PB, Drake JM, Rutka JT. Neurosurgical management of intracranial epidermoid tumors in children. Clinical article. J Neurosurg Pediatr 2009;4(2):91-96

3 Akahoshi M, Yanaka K, Watanabe D, et al. Atypical chronological changes on neuroimaging in the epidermoid in the frontal lobe with intracystic hemorrhage and tumor growth: case report. Radiol Case Rep 2018;13(6):1133-1136

4 Aribandi M, Wilson NJ. CT and MR imaging features of intracerebral epidermoid-a rare lesion. $\mathrm{Br} \mathrm{J}$ Radiol 2008;81(963):e97-e99

5 Berhouma M, Bahri K, Jemel H, Khaldi M. Intracerebral epidermoid tumor: pathogenesis of intraparenchymal location and magnetic resonance imaging findings. J Neuroradiol 2006;33(4):269-270

6 Chandler WF, Farhat SM, Pauli FJ. Intrathalamic epidermoid tumor. Case report. J Neurosurg 1975;43(5):614-617

7 Clark WC, Woodall W, Nicoll JA, Coakham HB. Intracerebral epithelial cyst: immunohistological diagnosis and endoneurosurgical treatment. Br J Neurosurg 1989;3(4):507-512

8 Diyora B, Nayak N, Kale D, Kamble H, Sharma A. Frontal intraparenchymal "white epidermoid cyst": a rare occurrence. J Neurosci Rural Pract 2010;1(2):125-127

9 Fawcitt RA, Isherwood I. Radiodiagnosis of intracranial pearly tumours with particular reference to the value of computer tomography. Neuroradiology 1976;11(5):235-242

10 Fox BD, Smitherman SM, Amhaz H, et al. A supratentorial, hemorrhagic, intraparenchymal epidermoid cyst. J Clin Neurosci 2009;16(8):1101-1105

11 Hanft SJ, Komotar RJ, Raper DM, , Sisti MB, McKhann GM II. Epidermoid tumors of the temporal lobe as epileptogenic foci. J Clin Neurosci 2011;18(10):1396-1399

12 Hu XY, Hu CH, Fang XM, Cui L, Zhang QH. Intraparenchymal epidermoid cysts in the brain: diagnostic value of MR diffusion-weighted imaging. Clin Radiol 2008;63(7):813-818

13 Iaconetta G, Carvalho GA, Vorkapic P, Samii M. Intracerebral epidermoid tumor: a case report and review of the literature. Surg Neurol 2001;55(4):218-222

14 Kaido T, Okazaki A, Kurokawa S, Tsukamoto M. Pathogenesis of intraparenchymal epidermoid cyst in the brain: a case report and review of the literature. Surg Neurol 2003;59(3):211-216

15 Kannan S, Kumar AS, Syamala S. A rare case of pediatric intraparenchymal epidermoid cyst: case report and review of literature. J Pediatr Neurosci 2018;13(1):96-99

16 von Koch CS, Young G, Chin CT, Lawton MT. Magnetic resonance imaging/spectroscopy of an intraaxial epidermoid: similarity to an abscess. Case illustration. J Neurosurg 2002;97(2):492-492
17 Lian K, Schwartz ML, Bilbao J, Perry J, Aviv RI, Symons SP. Rare frontal lobe intraparenchymal epidermoid cyst with atypical imaging. J Clin Neurosci 2012;19(8):1185-1187

18 de Macêdo Filho LJM, Aguiar GCM, Pessoa FC, et al. Intraparenchymal epidermoid cyst close to Broca area-awake craniotomy and gross total resection. World Neurosurg 2020;141:367-372

19 Mohanty S, Bhattacharya RN, Tandon SC, Shukla PK. Intracerebral cystic epidermoid. Report of two cases. Acta Neurochir (Wien) 1981;57(1-2):107-113

20 Nagaraj N, Bysani P, Dutta S, Nagesh S, Nandeesh B, Pai B. Rare case of atypical intraparenchymal epidermoid cyst. J Curr Res Sci Med 2018;4(1):68

21 Puranik A, Sankhe S, Goel N, Mahore A. Cerebral shading sign in a giant intraparenchymal white epidermoid. Neurol India 2012;60(2):265-266

22 Sener RN. Intraparenchymal epidermoid tumor of the brain. Eur Radiol 2005;15(4):838-839

23 Yan PX, Yu CJ. Minicraniotomy treatment of an intracerebral epidermoid cyst. Minim Invasive Neurosurg 2004;47(4):245-248

24 Zheng J, Wang C, Liu F. Intraparenchymal epidermoid cyst: proper surgical management may lead to satisfactory outcome. J Neurooncol 2018;138(3):591-599

25 Chambers AA, Lukin RR, Tomsick TA. Cranial epidermoid tumors: diagnosis by computed tomography. Neurosurgery 1977;1(3):276-280

26 Rengachary S, Kishore PR, Watanabe I. Intradiploic epidermoid cyst of the occipital bone with torcular obstruction. Case report. J Neurosurg 1978;48(3):475-478

27 Chen C-Y, Wong J-S, Hsieh S-C, Chu J-S, Chan WP. Intracranial epidermoid cyst with hemorrhage: MR imaging findings. AJNR Am J Neuroradiol 2006;27(2):427-429

28 Inoue Y, Ohata K, Nakayama K, Haba T, Shakudo M. An unusual middle fossa interdural epidermoid tumor. Case report. J Neurosurg 2001;95(5):902-904

29 Andica C, Hori M, Kamiya K, et al. Spatial restriction within intracranial epidermoid cysts observed using short diffusion-time diffusion-weighted imaging. Magn Reson Med Sci 2018;17(3):269-272

30 Johnston F, Crockard H. Dermoid, epidermoid and neurenteric cysts. Brain Tumors 1995;xx:895-905

31 Abramson RC, Morawetz RB, Schlitt M. Multiple complications from an intracranial epidermoid cyst: case report and literature review. Neurosurgery 1989;24(4):574-578

32 Patibandla MR, Yerramneni VK, Mudumba VS, Manisha N, Addagada GC. Brainstem epidermoid cyst: an update. Asian J Neurosurg 2016;11(3):194-200

33 Leal O, Miles J. Epidermoid cyst in the brain stem. Case report. J Neurosurg 1978;48(5):811-813

34 Sari A, Ozdemir O, Kosucu P, Ahmetoglu A. Intra-axial epidermoid cysts of the brainstem.J Neuroradiol 2005;32(4):283-284 